\title{
Guided osteoporotic bone regeneration with composite scaffolds of mineralized ECM/heparin membrane loaded with BMP2-related peptide
}

This article was published in the following Dove Press journal: International Journal of Nanomedicine

\author{
Tingfang Sun ${ }^{1, *}$ \\ Man Liu',* \\ Sheng Yao' \\ Yanhui Ji' \\ Lei $\mathrm{Shi}^{3}$ \\ Kai Tang' \\ Zekang Xiong' \\ Fan Yang \\ Kaifang Chen' \\ Xiaodong Guo \\ 'Department of Orthopedics, Union \\ Hospital, Tongji Medical College, \\ Huazhong University of Science and \\ Technology, Wuhan 430022, China; \\ ${ }^{2}$ Department of Gastroenterology and \\ Hepatology, Taikang Tongji Hospital, \\ Wuhan 430050, China; ${ }^{3}$ State Key \\ Laboratory of Materials Processing \\ and Die \& Mould Technology, School \\ of Materials Science and Engineering, \\ Huazhong University of Science and \\ Technology, Wuhan 430074, China \\ *These authors contributed equally \\ to this work
}

\begin{abstract}
Introduction: At present, the treatment of osteoporotic defects poses a great challenge to clinicians, owing to the lower regeneration capacity of the osteoporotic bone as compared with the normal bone. The guided bone regeneration (GBR) technology provides a promising strategy to cure osteoporotic defects using bioactive membranes. The decellularized matrix from the small intestinal submucosa (SIS) has gained popularity for its natural microenvironment, which induces cell response.
\end{abstract}

Materials and methods: In this study, we developed heparinized mineralized SIS loaded with bone morphogenetic protein 2 (BMP2)-related peptide P28 (mSIS/P28) as a novel GBR membrane for guided osteoporotic bone regeneration. These mSIS/P28 membranes were obtained through the mineralization of SIS (mSIS), followed by P28 loading onto heparinized $\mathrm{mSIS}$. The heparinized mSIS membrane was designed to improve the immobilization efficacy and facilitate controlled release of P28. P28 release from mSIS-heparin-P28 and its effects on the proliferation, viability, and osteogenic differentiation of bone marrow stromal stem cells from ovariectomized rats (rBMSCs-OVX) were investigated in vitro. Furthermore, a critical-sized OVX calvarial defect model was used to assess the bone regeneration capability of mSIS-heparin-P28 in vivo.

Results: In vitro results showed that P28 release from mSIS-heparin-P28 occurred in a controlled manner, with a long-term release time of 40 days. Moreover, mSIS-heparin-P28 promoted cell proliferation and viability, alkaline phosphatase activity, and mRNA expression of osteogenesis-related genes in rBMSCs-OVX without the addition of extra osteogenic components. In vivo experiments revealed that mSIS-heparin-P28 dramatically stimulated osteoporotic bone regeneration.

Conclusion: The heparinized mSIS loaded with P28 may serve as a potential GBR membrane for repairing osteoporotic defects.

Keywords: osteoporotic defect, guided bone regeneration, decellularized matrix, heparin, BMP2-related peptide, control release

\section{Introduction}

Osteoporosis, an emerging health care issue worldwide, is characterized by the reduction in bone mass, poor bone strength, and microarchitectural deterioration, leading to the risk of skeletal fragility and increased susceptibility to fractures. ${ }^{1}$ The currently available strategies for the prevention and treatment of osteoporosis involve the use of agents that inhibit the osteoclast activity and, hence, bone resorption. These regimens are incapable of regenerating an osteoporotic bone, especially in osteoporotic defects. ${ }^{2}$ Guided bone regeneration (GBR) is a generally accepted therapeutic
Correspondence: Xiaodong Guo Department of Orthopedics, Union Hospital, Tongji Medical College, Huazhong University of Science and Technology, 1277 Jiefang Avenue, Wuhan 430022, China

Tel +86 I 53272 I 6660

Email xiaodongguo@hust.edu.cn 
modality used to achieve bone regeneration ${ }^{3}$ and may serve as a promising strategy to cure osteoporotic fractures.

GBR is based on the application of a barrier membrane that covers an osseous defect and excludes any interference from nonosteogenic tissues in the process of bone healing, while providing a favorable environment for bone regeneration. ${ }^{4}$ Thus, an excellent barrier membrane is indispensable for GBR surgery under clinical settings. ${ }^{5}$ The decellularized matrix from the small intestinal submucosa (SIS) has attracted the attention of researchers, as it highly retains the microstructure and composition of the natural extracellular matrix (ECM), which provides biologic cues to regulate the adhesion, proliferation, migration, and differentiation of stem cells. ${ }^{6}$ SIS comprises $\sim 90 \%$ collagen (mainly type I) ${ }^{7}$ and smaller amounts of glycosaminoglycans, ${ }^{8}$ glycoproteins, and growth factors such as vascular endothelial growth factor and fibroblast growth factor 2.9,10 The pure SIS membrane was used as a GBR membrane and shown to support a pro-osteogenic remodeling microenvironment in vivo. ${ }^{4,11}$ However, its osteoinductive capability is far from that required to induce satisfactory regeneration of the osteoporotic bone, wherein the regeneration capability is lower than that in normal bones.

Biomineralization is a recognized treatment to improve the osteogenic ability of collagen-based scaffolds. ${ }^{12}$ The mineralization of collagen-based scaffolds is shown to result in a chemical composition of collagen/apatite similar to that of the natural bone and support cell adhesion, proliferation, and osteogenic differentiation. ${ }^{13,14}$ Collagen is a good template for the apatite deposition, growth, and nucleation, whereas noncollagenous proteins allow the formation of a hierarchically staggered nanostructure of mineralized collagen that provides a broad range of mechanical functionality and excellent biologic performance. ${ }^{15}$ Therefore, SIS was considered to be an excellent template for biomineralization as an ECM-based scaffold composed of collagen and noncollagenous proteins.

Another strategy to enhance the osteoinductive capacity involves the incorporation of growth factors into the scaffold. Bone morphogenetic protein 2 (BMP2), a member of the transforming growth factor $\beta$ superfamily, is most extensively studied for its ability to induce bone regeneration in tissue engineering. ${ }^{16}$ However, BMP2 poses some challenges such as short half-life, risk of ectopic bone formation, and tumorigenesis due to off-target effects. ${ }^{17}$ To overcome these drawbacks, a short peptide, P28 ( $\mathrm{S}^{[\mathrm{PO} 4]}$ DDDDDDDKIPKASSVPTELSAISTLYL, molecular weight: 3,091.20), derived from the knuckle epitope of BMP2, was designed by our team in a previous study. ${ }^{18}$
Aside from its binding to the BMP receptor through its core domain, this peptide possesses a short block of seven aspartic acid (Asp) residues that confers high affinity for bone and hydroxyapatite (HA), thereby achieving a bone-targeted effect and controlled delivery from HA-containing materials, respectively. ${ }^{17,19,20} \mathrm{We}$ confirmed that $\mathrm{P} 28$ promotes the repair of calvarial defects in a normal rat. ${ }^{17,18}$ However, whether P28 may induce satisfactory bone regeneration under osteoporotic pathologic conditions is questionable.

The optimization of the biologic effect of P28 necessitates the development of a smart delivery system, which allows local P28 release at sufficient concentrations for extended time. The glycosaminoglycan heparin, a highly sulfated and anionic linear polysaccharide, is known to specifically bind with several growth factors, cytokines, chemokines, and other signaling molecules as well as the proteins of the ECM. ${ }^{21,22}$ Several studies have shown that the incorporation of heparin chains into collagen, calcium phosphate scaffolds, and fibrin gel increases their growth factor-loading capacity and facilitates controlled release. ${ }^{23}$ Based on these natural binding mechanisms, the design of biomaterials with drug delivery properties has proved to be a meaningful strategy. SIS as an ECM provides considerable amounts of bioactive sites for heparin modification. In this study, we tried to modify mineralized SIS (mSIS) with heparin to facilitate the binding and controlled local release of P28.

Although several studies have investigated GBR technology in healthy bone, very few reports have explored its applications in the osteoporotic bone. Here, we aimed to develop a novel GBR membrane, heparinized mSIS loaded with P28 (mSIS/P28), for guided osteoporotic bone regeneration. The release profile of P28 from mSIS-heparin-P28 was investigated in vitro. We hypothesize that a smart delivery system for mSIS-heparin-P28 may facilitate the local and sustained release of P28 and the combination of mSIS and P28 may synergistically enhance osteoporotic bone regeneration to stimulate osteogenesis under osteoporotic pathologic conditions both in vivo and in vitro.

\section{Materials and methods Preparation of SIS and mSIS}

Porcine small intestine was harvested from healthy homeraised pigs (around $100 \mathrm{~kg}$ at 6 months) within $3 \mathrm{~h}$ of sacrifice. The decellularized SIS matrix was prepared as previously described. ${ }^{24}$ In brief, porcine jejunum was sequentially subjected to mechanical dissociation, degreasing, enzyme digestion, detergent, lyophilization, and sterilization. To obtain mSIS, SIS membranes $\left(20 \times 20 \times 0.5 \mathrm{~mm}^{3} ; 5 \times 5 \times 0.5 \mathrm{~mm}^{3}\right)$ were 
soaked in $50 \mathrm{~mL}$ simulated body fluid (SBF) at $37^{\circ} \mathrm{C}$ for 7 and 14 days; SBF solution was refreshed every 2 days. ${ }^{25}$ At predetermined intervals, the specimens were washed with an excess of deionized water (10 min each for six times) to remove any soluble inorganic ions and freeze-dried for further characterization and use.

\section{Preparation of heparinized $\mathrm{mSIS}$ and $\mathrm{mSIS} / \mathrm{P} 28$}

Heparinized mSIS was prepared as previously described. ${ }^{23,26}$ SIS incubated in the SBF solution for 14 days was used for this study. mSIS was functionalized with heparin by coupling via $N$-(3-dimethylaminopropyl)- $N^{\prime}$-ethylcarbodiimide hydrochloride and $N$-hydroxysuccinimide chemistry at a ratio of $75 \mathrm{mg}$ heparin (molecular weight: 4,000-6,000; SigmaAldrich, St Louis, MO, USA) per $1 \mathrm{~g}$ mSIS.

Heparinized or pure mSIS without any modification $\left(20 \times 20 \times 0.5 \mathrm{~mm}^{3} ; 5 \times 5 \times 0.5 \mathrm{~mm}^{3}\right)$ was incubated with $1 \mathrm{~mL}$ aqueous bovine serum albumin solution (5\%) containing $3 \mathrm{mg} \mathrm{P} 28$ for $24 \mathrm{~h}$ at $37^{\circ} \mathrm{C}$ in a humidified atmosphere. The unreacted P28 peptides were washed with double-distilled water (three washes for $5 \mathrm{~min}$ each). The membranes were lyophilized and sterilized with ethylene oxide gas. The mSISheparin-P28 membrane was simply referred to as mSIS/P28, unless otherwise stated.

\section{Characterization of SIS and mSIS Histology analysis}

For histologic analysis, SIS was fixed in 4\% paraformaldehyde and embedded in paraffin. Paraffin sections were stained with $\mathrm{H} \& \mathrm{E}$ to observe the extent of cellular remnants and the structure of collagen fibers under a microscope (Olympus IX71; Olympus Corporation, Tokyo, Japan).

\section{Scanning electron microscopy}

The superficial microstructure of SIS or mSIS was characterized by scanning electron microscopy (SEM; Sirion 200; FEI Co., Eindhoven, the Nertherlands). Samples were sputter-coated with gold before scanning. The operated voltage was set at $15 \mathrm{kV}$.

\section{Energy-dispersive spectrometry and X-ray diffraction} The elements of mSIS were determined with SEM fitted with an energy-dispersive X-ray spectrometer (EDS). The crystalline phases in SIS before and after immersion in SBF for 7 and 14 days were examined by X-ray diffraction (XRD, X'Pert PRO; PANalytical B.V., Almelo, the Netherlands) using $\mathrm{CuK}$ alpha radiation $(\lambda=0.15406 \mathrm{~nm})$ at a scanning rate of $1 \%$ min in the range of $10^{\circ}-80^{\circ}$.

\section{Immobilization efficacy of P28 on membranes and its release kinetics}

The molar content of P28 immobilized onto mSIS $\left(5 \times 5 \times 0.5 \mathrm{~mm}^{3}\right)$ was calculated from the difference between the primary feed molarity and actual washing out and residual molar content. In addition, P28 content was determined using high-performance liquid chromatography, as previously described. ${ }^{27}$

To investigate the release kinetics of P28, mSIS/P28 $\left(5 \times 5 \times 0.5 \mathrm{~mm}^{3}\right)$ with or without heparinization was incubated in $200 \mathrm{~mL}$ PBS at $37^{\circ} \mathrm{C}$ for 14 days. The eluted P28 peptide was quantified at day $0.5,1,3,5,7,10,14,17,21$, $25,29,33$, and 37 following incubation. At the predetermined time point, the supernatant was completely collected and refreshed with PBS. The amount of P28 peptide in the collected supernatant was measured by high-performance liquid chromatography.

\section{Establishment of the osteoporosis model}

All animal procedures were approved by the Animal Ethics Committee of Huazhong University of Science and Technology (HUST). Three-month-old female Sprague Dawley rats were purchased from the Tongji Experimental Animals Center, Tongji Medical College of HUST and the experiments performed according to the recommendations of the Guide for the Care and Use of Laboratory Animals of the National Institutes of Health. To generate the osteoporosis model, rats were subjected to bilateral ovariectomy (OVX) or sham operation (Sham). Three months after ovariectomy, the femurs were explanted from OVX and Sham groups and scanned on a micro-computed tomography (micro-CT) imaging system (SkyScan 1176 X-ray microtomography; Bruker, Billerica, MA, USA). Sections in the femoral condyle were chosen and a series of slices starting at a distance of $1 \mathrm{~mm}$ proximal to the end of the growth plate with a length of $2 \mathrm{~mm}$ were selected for evaluation. The following parameters were analyzed for each group $(n=3)$ using the CT-analyzer software: bone volume fraction (bone volume [BV]/tissue volume $[\mathrm{TV}])$, trabecular number (Tb.N), trabecular thickness (Tb.Th), and trabecular separation (Tb.Sp).

\section{Isolation and characterization of bone marrow stromal stem cells from ovariectomized rats (rBMSCs-OVX)}

Primary rBMSCs-OVX were established as previously described. ${ }^{28,29}$ After induction for 3 months, OVX rats were sacrificed by an overdose of pentobarbital sodium. Bone 
marrow was harvested from the femur, and rBMSCs-OVX were isolated by rapid plastic adhesion of the bone marrowderived cells. The growth medium for rBMSCs-OVX comprised DMEM/F12 (Thermo Fisher Scientific, Waltham, MA, USA) supplemented with 10\% fetal bovine serum (Thermo Fisher Scientific) and 1\% penicillin/streptomycin. The cells were cultured in $25 \mathrm{~cm}^{2}$ culture flasks under a humidified atmosphere containing $5 \% \mathrm{CO}_{2}$ at $37^{\circ} \mathrm{C}$. rBMSCs from the Sham group were similarly isolated (rBMSCs-Sham).

To evaluate the differences between the osteogenic differentiation capacity of rBMSCs-OVX and rBMSCs-Sham, Alizarin Red S (ARS) staining was performed. Briefly, rBMSCs-OVX or rBMSCs-Sham $\left(1 \times 10^{5}\right.$ cells $)$ were seeded on six-well tissue culture plates and cultured in osteogenic media comprising DMEM (HyClone, Logan, UT, USA) supplemented with $10 \%$ fetal bovine serum, $10 \mathrm{mM}$ sodium $\beta$-glycerol phosphate, $50 \mu \mathrm{g} / \mathrm{mL}$ L-ascorbic acid, and $10^{-8} \mathrm{M}$ dexamethasone (Sigma-Aldrich) for 14 and 21 days. ARS staining was performed according to a previous study. ${ }^{27}$ The optical density of Alizarin Red extracted from the samples was spectrophotometrically determined at $405 \mathrm{~nm}$ wavelength using a microplate reader.

\section{Proliferation of rBMSCs-OVX on SIS, $\mathrm{mSIS}$, and $\mathrm{mSIS} / \mathrm{P} 28$}

The cell proliferation on SIS, mSIS, and mSIS/P28 membranes was evaluated with MTT colorimetric assay. Briefly, rBMSCs-OVX were seeded on SIS, mSIS, and mSIS/P28 membranes $\left(20 \times 20 \times 0.5 \mathrm{~mm}^{3}\right)$ in six-well tissue culture plates at $2 \times 10^{5}$ cells/well and cultured in the growth medium. MTT assay was performed in triplicates for each group after the cells were cultured for 1, 3, 5, and 7 days. At each time point, the growth medium was removed and the cells/scaffolds were rinsed with PBS and incubated in MTT solution for $3 \mathrm{~h}$ at $37^{\circ} \mathrm{C}$. The formazan crystals were dissolved in dimethyl sulfoxide, and $100 \mathrm{~mL}$ of the aspirate was transferred into a 96-well plate to measure its absorbance at $540 \mathrm{~nm}$ wavelength. MTT assay was also performed for scaffolds without seeded cells and used as the control. The results are shown as units of the absorbance value.

Calcein-AM/propidium iodide double staining (live/dead assay; Sigma-Aldrich) was used to quantify cell viability. Cells were cultured on membranes for 3 and 14 days and live/dead staining was performed according to the manufacturer's protocol. Images were obtained using a multi-photon confocal fluorescence microscope equipped with a digital camera (Nikon Corporation, Tokyo, Japan). Image-Pro Plus software 6.0 (Media Cybernetics, Carlsbad, CA, USA) was used to quantify the percentage of live cells according to our previous study. ${ }^{18}$

\section{Alkaline phosphatase (ALP) staining and quantitative analyses}

ALP is an early osteogenic differentiation marker. rBMSCsOVX $\left(2 \times 10^{5}\right.$ cells $)$ were seeded on SIS, mSIS, and mSIS/ P28 membranes and cultured in the growth medium (without osteogenic components) for 7 and 14 days. ALP staining was performed using 5-bromo-4-chloro-3-indolyl phosphate/ nitro blue tetrazolium color development kit (Beyotime Biotechnology, Nanjing, China) at predetermined time points. ALP activity was quantitatively analyzed using a SensoLyte pNPP ALP assay kit (Beyotime Biotechnology). The double-stranded DNA concentration in each sample was quantified by PicoGreen ${ }^{\circledR}$ dsDNA assay kit (Thermo Fisher Scientific), and the ALP activity of each sample was normalized to dsDNA concentration. All experiments were performed in triplicates.

\section{Osteogenesis-related gene expression}

We seeded rBMSCs-OVX $\left(2 \times 10^{5}\right.$ cells/membrane $)$ on SIS, $\mathrm{mSIS}$, and $\mathrm{mSIS} / \mathrm{P} 28$ membranes in the presence of the growth medium (without osteogenic components) for 7 and 14 days. Total RNA of each sample was isolated using Trizol reagent (Thermo Fisher Scientific) and cDNA synthesized using Prime-Script ${ }^{\mathrm{TM}}$ RT reagent kit (Takara Bio, Shiga, Japan) according to the manufacturer's recommendations. Real-time polymerase chain reaction was performed on a Step One Real-Time polymerase chain reaction system (Thermo Fisher Scientific) using the cDNA template, specific primers, and SYBR Green supermix (Takara Bio). The primers for the selected genes are shown in Table 1. All experiments were performed in triplicates.

Table I PCR primers for genes encoding Runx2, Col-I $\alpha$, Ocn, Opn, and GAPDH

\begin{tabular}{ll}
\hline Gene & Primer sequence \\
\hline GAPDH & F5'-GACAAAATGGTGAAGGTCGGT \\
& R5'-GAGGTCAATGAAGGGGTCG \\
Runx2 & F5'-AACTTGCTAACGTGAATGGTC \\
& R5'-TAGCCCACTGAAGAAACTTGG \\
Col-l $\alpha$ & F5'-CTGAGATGCTCCCTAGACC \\
& R5'-CCCTTGTTAAATAGCACCTTC \\
Ocn & F5'-GAACAGACAAGTCCCACACAG \\
& R5'-TCAGCAGAGTGAGCAGAAAGAT \\
Opn & F5'-GAGGTGATAGCTTGGCTTACGG \\
& R5'-ACGCTGGGCAACTGGGAT \\
\hline
\end{tabular}

Abbreviation: $\mathrm{PCR}$, polymerase chain reaction. 


\section{Implantation of SIS, mSIS, and mSIS/ P28 membranes in calvarial defects of OVX rats}

All procedures involving animals were performed according to the National Institutes of Health Animal Care Guidelines. The critical-sized cranial defect experiment in rats was approved by the Animal Ethics Committee of HUST. Fifteen OVX rats from the established osteoporosis model were randomly divided into the following three groups (five rats per group): SIS, mSIS, and $\mathrm{mSIS} / \mathrm{P} 28$. The rats were anesthetized with an intraperitoneal injection of sodium pentobarbital ( $40 \mathrm{mg} / \mathrm{kg}$ body weight). A $1.5 \mathrm{~cm}$ sagittal incision was made on the scalp and a critical-sized defect was created using a trephine burr of $5 \mathrm{~mm}$ diameter. The calvarial defects were filled with SIS, mSIS, and mSIS/P28 membranes $\left(5 \times 5 \times 0.5 \mathrm{~mm}^{3}\right)$. After 8 weeks of surgery, the animals were sacrificed with an overdose of pentobarbital administered intraperitoneally and all harvested craniums were fixed in $4 \%$ paraformaldehyde before further analysis.

\section{Micro-CT analysis of bone defects}

The harvested specimens were scanned on a micro-CT scanner (SkyScan $1176 \mathrm{X}$-ray microtomography; Bruker) at a resolution of $18 \mu \mathrm{m}$ to evaluate the bone regeneration in the cranium defect. After three-dimensional reconstruction, the bone mineral density (BMD) and the bone volume fraction $(\mathrm{BV} / \mathrm{TV})$ in the defect regions were used to calculate the new bone formation using $\mathrm{CT}$ analysis software. ${ }^{30}$

\section{Histologic analysis}

Specimens were decalcified, dehydrated, and embedded in paraffin blocks. Sagittal sections $(5 \mu \mathrm{m})$ of the central segment were prepared and stained with H\&E. Morphometric analysis of H\&E-stained sections from each group $(n=5)$ was performed using Image-Pro Plus software to assess the new bone fraction and the new vessel density in the defect. New bone fraction was calculated by dividing the new bone area with the total defect area. New vessel density was calculated by dividing the new vessel numbers with the total defect area.

\section{Statistical analysis}

All experiments were performed in triplicate data sets unless specified otherwise. All data are presented as mean \pm SD. Statistical significance was tested using analysis of variance followed by Tukey's post hoc test. A value of $p<0.05$ (confidence level of 95\%) was considered as statistically significant.

\section{Results Histologic analysis and SEM observation of the extracted SIS}

As shown in Figure 1, H\&E staining revealed the presence of multiple collagen fibers in the SIS membrane with no visible cellular component, indicative of complete decellularization. SEM examination further verified the presence of a network of nanoscale fibers in SIS with relatively sufficient decellularization (Figure 1D and G).

The process of incubating scaffolds in SBF solution is universally recognized as the method for scaffold mineralization..$^{31,32}$ The superficial structure of the SIS membrane was characterized by SEM for the detection of mineralized crystals. As shown in Figure 1, crystal formation was observed on the surface of collagen fibers on days 7 and 14 . The density and particle size of crystals increased from days 7 to 14 . The crystal size ranged from 10 to $900 \mathrm{~nm}$. The elemental composition of these crystals was determined by EDS (Figure 2A). The EDS results showed that $\mathrm{Ca} / \mathrm{P}$ ratio was 1.76 , which is close to 1.67 , the $\mathrm{Ca} / \mathrm{P}$ ratio of $\mathrm{HA}$ in natural bone. ${ }^{33}$ The crystallographic structure of the crystals on mSIS was investigated by XRD. Figure $2 \mathrm{~B}$ displays the XRD pattern of crystals on mSIS with sharp peaks, indicative of good crystallinity. In addition, two characteristic peaks of (211) and (203) for HA (XRD JCPDS data file no 09-0432) were distinct for SIS incubated with SBF for 7 and 14 days, but not for pure SIS. Thus, HA was successfully deposited on SIS. Moreover, the two peaks became stronger with prolonged mineralization time, suggesting that the particle size of crystals on SIS was greater at 14 days than that observed at 7 days.

\section{In vitro release kinetics of $\mathrm{P} 28$ peptide}

When the heparin-conjugated mSIS membrane was immersed in P28 solution, the efficiency of P28 immobilization onto the membrane was up to $86.32 \% \pm 4.27 \%(2.50 \pm 0.13 \mathrm{mg} /$ membrane $\left.\left[5 \times 5 \times 0.5 \mathrm{~mm}^{3}\right]\right)$. However, the efficiency of P 28 immobilization onto pure mSIS without heparinization was only $62.15 \% \pm 3.76 \%$. Thus, heparinization of mSIS resulted in a significant improvement in P28 immobilization efficiency as compared with pure mSIS $(p<0.05)$.

The release kinetics of P28 peptide from mSIS/P28 with or without heparin binding are shown in Figure 3. After a minor burst release (15.33\%), P28 release from mSIS/P28 in the presence of heparin occurred in a steady and sustained manner. By day $40, \sim 94.13 \%$ of total P28 peptide was released. In contrast, the release curve of $\mathrm{P} 28$ from pure mSIS showed a burst release on day $1(25.87 \%)$ and near-complete 

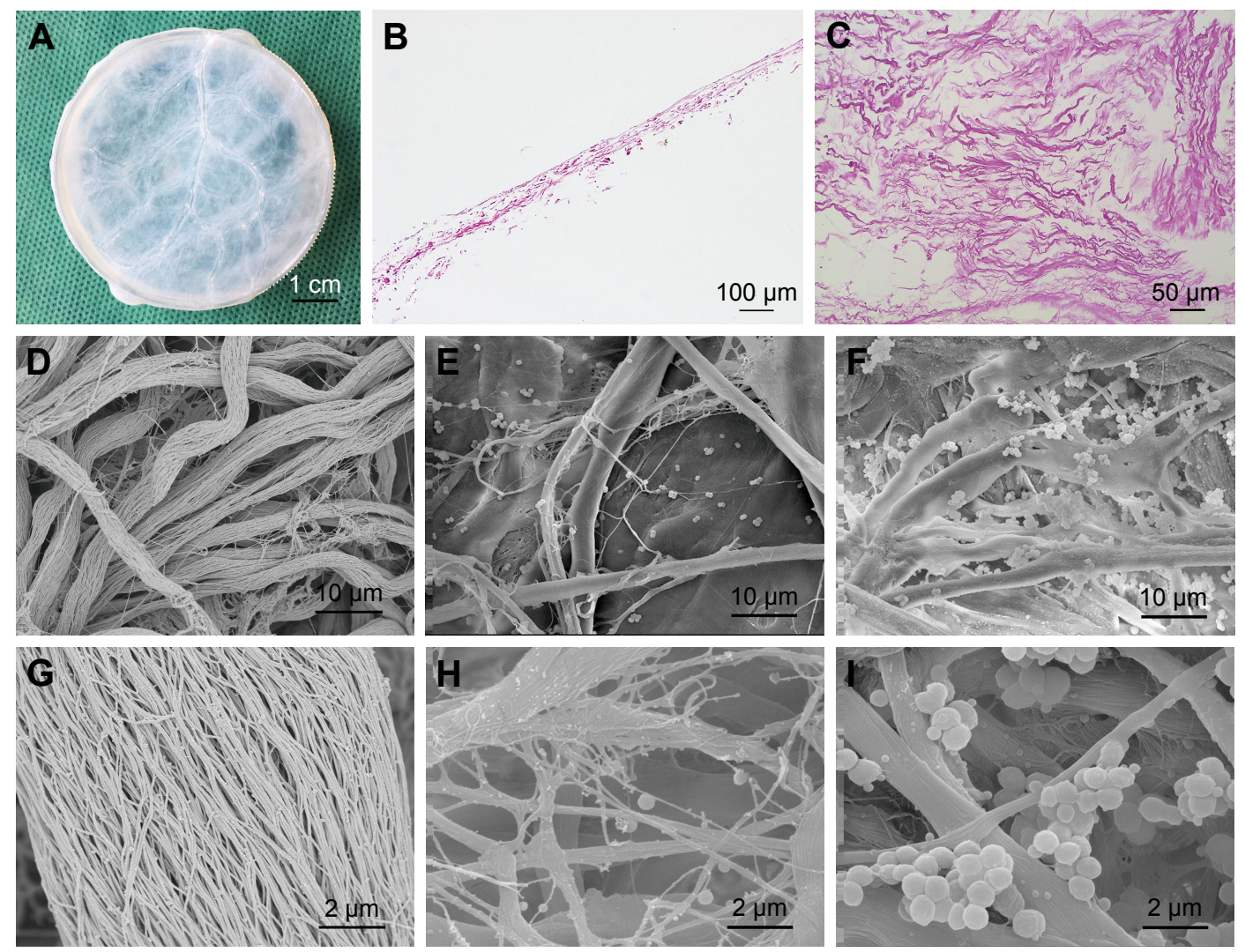

Figure I Structural properties of the scaffolds.

Notes: (A) Macroscopic view of SIS membrane after decellularization treatment. (B) H\&E staining image of SIS sectioned at longitudinal direction. (C) H\&E staining image of SIS sectioned at horizontal direction. (D-I) SEM images of SIS incubated in SBF solution for 0 days (D and $\mathbf{G}), 7$ days (E and $\mathbf{H})$, and I4 days (F and I) at two magnifications. Abbreviations: SBF, simulated body fluid; SEM, scanning electron microscopy; SIS, small intestinal submucosa.

release (98.35\%) by day 21 (b in Figure 3). Thus, the heparinfunctionalized mSIS showed a more controlled release process as compared with pure mSIS without heparin treatment. Therefore, heparin-functionalized mSIS loaded with P28 (mSIS/P28) was selected for the subsequent study.

\section{Reduced osteogenic potential in the osteoporosis model and rBMSCs-OVX}

As expected, micro-CT images from OVX rats presented a significant decrease in the trabecular BV and microstructure (Figure 4A), as previously observed. ${ }^{34}$ The values of BV/TV,
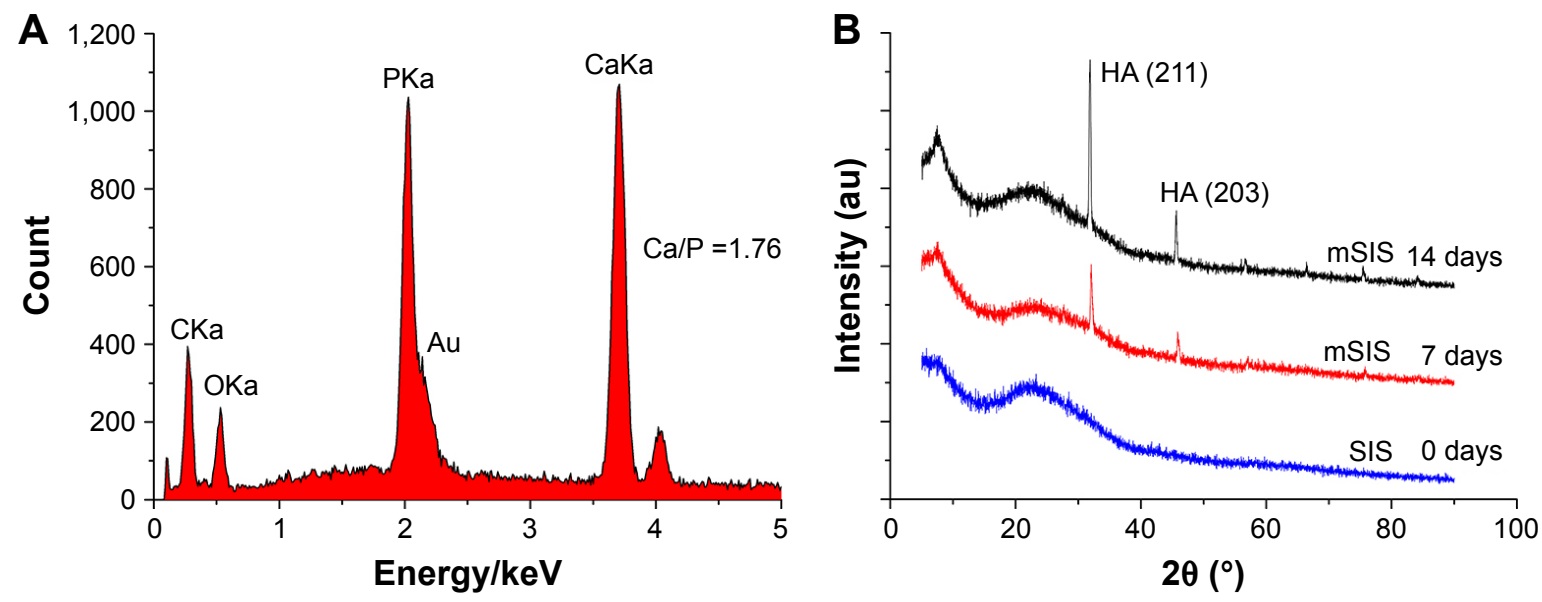

Figure 2 (A) EDS analysis of SIS membrane incubated in SBF for 7 days. The peak for gold (Au) in the EDS analysis comes from the gold sputtering coat. (B) XRD patterns of SIS membranes incubated in SBF for 0,7 , and 14 days.

Abbreviations: EDS, energy-dispersive X-ray spectrometer; HA, hydroxyapatite; mSIS, mineralized SIS; SBF, simulated body fluid; SIS, small intestinal submucosa; XRD, X-ray diffraction. 


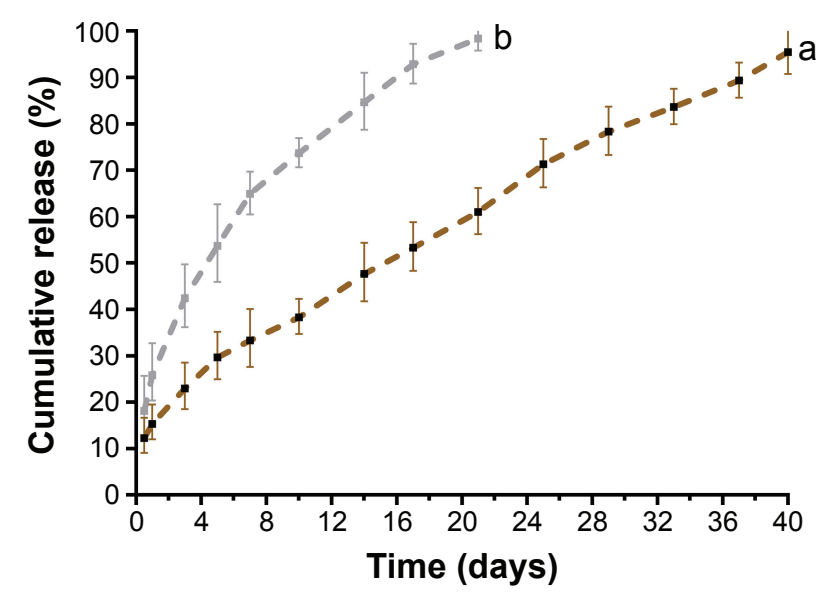

Figure 3 The profiles of P28 peptide released from mSIS in the (a) presence and (b) absence of heparin.

Abbreviation: $\mathrm{mSIS}$, mineralized SIS.

Tb.N, and Tb.Th were significantly lower in OVX group as compared with Sham group, while those of Tb.Sp were significantly higher in OVX rats as compared with Shamoperated rats $(p<0.05)$.

Calcium nodule deposition is a biomarker of osteogenic differentiation. To quantify the formation of extracellular calcium nodules, ARS staining was performed after osteogenic induction for 14 and 21 days. As shown in Figure 4B, nodule formation was observed in both groups, but the level of matrix mineralization was significantly lower in rBMSCs-OVX group as compared with rBMSCs-Sham group $(p<0.05)$. These results confirmed successful establishment of the osteoporosis model.

\section{Proliferation and viability of rBMSCs- OVX on SIS, mSIS, and mSIS/P28 membranes}

The amount of formazan dye corresponds to the level of active cellular metabolism, which directly correlates with the number of viable cells. As shown in Figure 5, the proliferation rate of rBMSCs-OVX was significantly higher on mSIS as compared with pure SIS $(p<0.05)$, indicating that the mSIS promoted rBMSCs-OVX proliferation. Furthermore, rBMSCs-OVX growing on P28-loaded mSIS exhibited enhanced proliferation as compared with those growing on pure SIS. Thus, mSIS/P28 membranes supported maximum cell proliferation.

The live/dead assay results for rBMSCs-OVX seeded on SIS, mSIS, and mSIS/P28 membranes are shown in Figure 6. Live cells stained with calcein-AM (green) adhered to membranes and showed normal spindle morphology, while dead cells stained with propidium iodide (red) were relatively few (Figure 6A). The density of live cells on all three membranes
(Figure 6B) increased with prolonged culture duration, supporting the cell proliferation data (Figure 5). The percentage of live cells (Figure 6C) was around 95\% and only minor variations were observed between the groups. The density of live cells was significantly higher on mSIS and mSIS/P28 membranes as compared with SIS membrane on days 7 and $14(p<0.05)$, with the highest density reported for $\mathrm{mSIS} / 28$ membrane.

\section{Osteogenic differentiation of rBMSCs- OVX on SIS, mSIS, and mSIS/P28 membranes}

We performed ALP staining of cells cultured on SIS, mSIS and $\mathrm{mSIS} / \mathrm{P} 28$ for 7 and 14 days (Figure 7A) and found that $\mathrm{mSIS}$ and $\mathrm{mSIS} / \mathrm{P} 28$ distinctly promoted ALP production as compared with SIS membrane, indicated by a more intense color reaction. The most intense color reaction was observed for mSIS/P28 group. Quantitative analysis revealed that ALP activity of cultured cells increased over time throughout the assay period (Figure 7B) and was significantly enhanced in $\mathrm{mSIS}$ and $\mathrm{mSIS} / \mathrm{P} 28$ groups as compared with SIS group $(p<0.05)$. Moreover, ALP activity for rBMSCs-OVX was significantly higher when cultured on $\mathrm{mSIS} / \mathrm{P} 28$ membrane as compared with mSIS membrane $(p<0.05)$.

The expression levels of osteogenic genes such as Runx2, Col-I $\alpha$, Ocn, and Opn were examined in SIS, mSIS, and mSIS/P28 groups after 7 and 14 days of cell culture. As an osteogenic transcription factor, Runx 2 mRNA showed a gradual decrease in the expression from days 7 to 14 (Figure 8). Among the three groups, mSIS/P28 group showed the highest expression of Runx 2 mRNA, demonstrating osteogenic induction at the early stage of the culture. The mRNA expression of bone ECM proteins ( $\mathrm{Col}-\mathrm{I} \alpha, \mathrm{Ocn}$, and $\mathrm{Opn}$ ) showed a gradual increase in mSIS and mSIS/P28 groups over time, indicative of matrix mineralization at the later stage. As the most sensitive markers of osteogenic differentiation, Ocn and Opn mRNA showed $>10$-fold increased expression in mSIS and $\mathrm{mSIS} / \mathrm{P} 28$ groups as compared with pure SIS group at day 14. Taken together, rBMSCs-OVX cultured on mSIS and $\mathrm{mSIS} / \mathrm{P} 28$ showed a significant increase in the expression of Runx2, Col-I $\alpha, O c n$, and Opn genes as compared with those cultured on pure SIS $(p<0.05)$. The highest level of expression was reported for mSIS/P28 group $(p<0.05)$.

\section{Osteoporotic bone regeneration in critical-sized rat calvarial defects}

\section{Micro-CT evaluation}

The representative micro-CT images of the rat calvarial are shown in Figure 9A. The control group (pure SIS) remained 

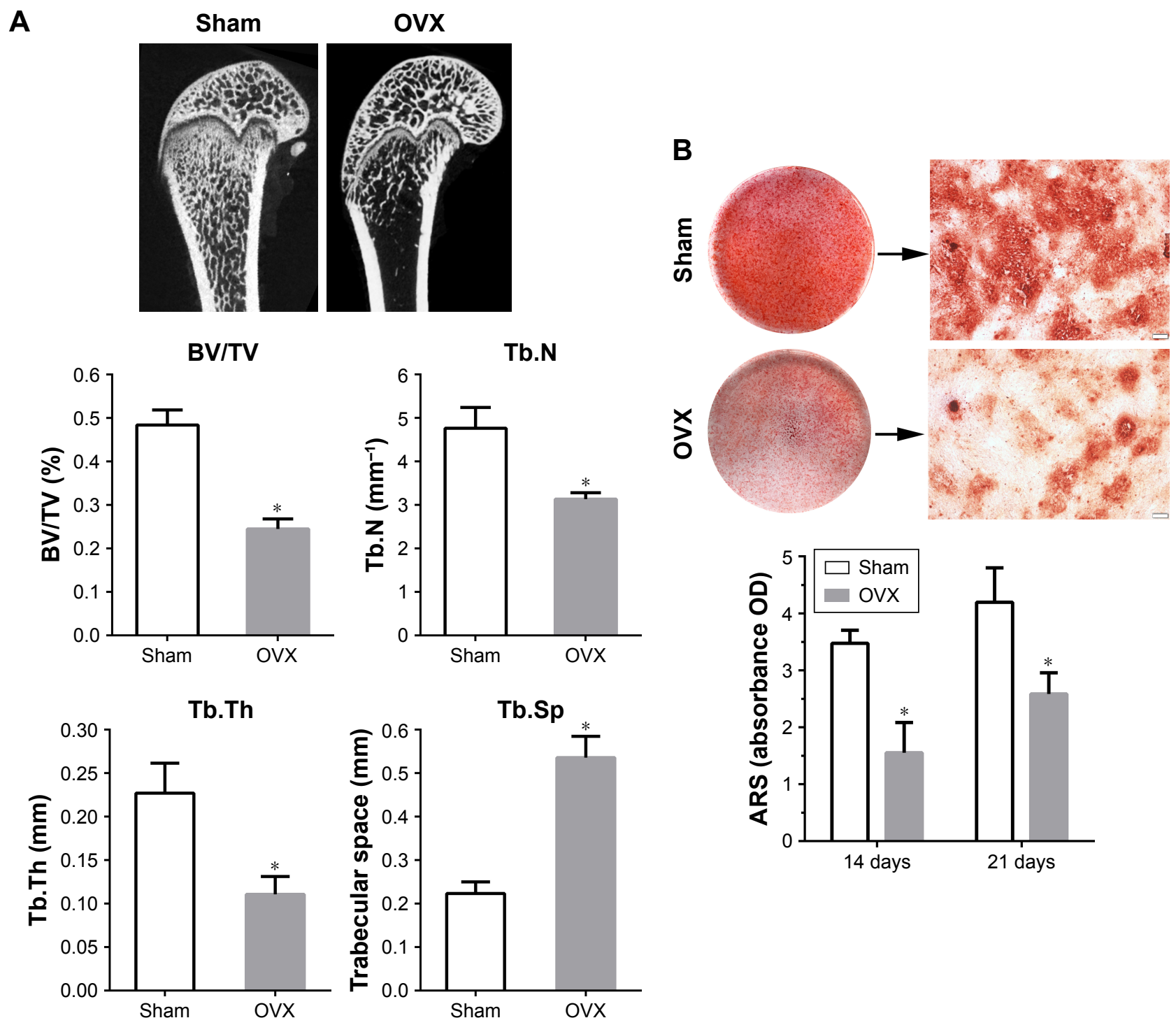

Figure 4 Establishment of OVX model.

Notes: (A) Micro-CT images of distal femoral shafts in Sham and OVX rats, and the morphometric analysis of the bone volume fraction (BV/TV), Tb.N, Tb.Th, and Tb.Sp in femoral shafts between Sham and OVX groups ( $n=5$ per group). (B) Alizarin Red staining for Sham and OVX rats-derived BMSCs after culturing in osteogenic medium for 21 days, and the quantitative analysis of mineralized nodules between Sham and OVX groups at days 14 and 21 . Statistical significance is indicated by $* p<0.05$ compared to Sham group. Magnification: 40x. Scale bar: $200 \mu \mathrm{m}$.

Abbreviations: ARS, Alizarin Red S; BMSC, bone marrow stromal stem cells; BV, bone volume; micro-CT, micro-computed tomography; OVX, ovariectomy; Tb.N, trabecular number; Tb.Sp, trabecular separation; Tb.Th, trabecular thickness; TV, tissue volume.

largely open and showed minimally mineralized regions at the edges of the defect. An evident increase was observed in mineralized tissues in defects implanted with mSIS as compared with those implanted with pure SIS scaffolds. Furthermore, the incorporation of P28 into mSIS resulted in the most evident calcification in the bone defects. The bone volume fraction (BV/TV, \%) for mSIS/P28 was almost double the value observed for mSIS $(p<0.05)$ and more than seven times the value obtained for control $(p<0.05$; Figure 9B). The density of the calcified tissue indicated by BMD value was quantitatively analyzed. Consistent with $\mathrm{BV} / \mathrm{TV}$ analysis, BMD result showed that mSIS significantly enhanced the density of the calcified tissue as compared to pure SIS and control $(p<0.05)$. Furthermore, mSIS/P28 significantly increased the density of the calcified tissue as compared with mSIS ( $p<0.05$; Figure 9C).

\section{Histologic analysis}

The images of H\&E-stained sections are shown in Figure 9D and F. No signs of inflammation or immunologic response were observed in all specimens. Histologic analysis confirmed the bone formation observed in micro-CT scans. The bone defect implanted with pure SIS membrane was filled with a thin connective fibrous tissue layer at 8 weeks and no 


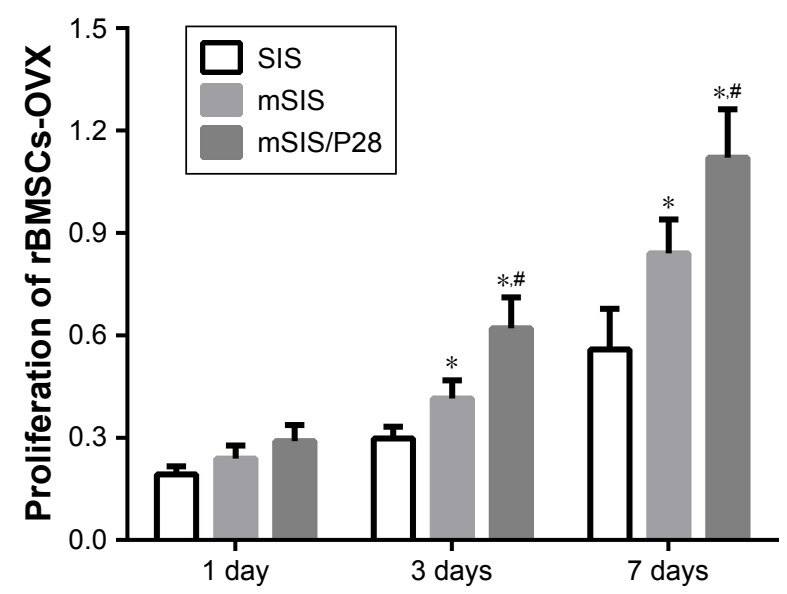

Figure 5 Proliferation of rBMSCs-OVX cells on SIS, mSIS, and mSIS/P28 membranes. Note: Statistical significance is indicated by $* p<0.05$ compared to pure SIS group and ${ }^{\#} p<0.05$ compared to mSIS group.

Abbreviations: mSIS, mineralized SIS; OVX, ovariectomy; rBMSCs-OVX, bone marrow stromal stem cells from ovariectomized rats; SIS, small intestinal submucosa.

apparent signs of bone formation were observed. In contrast, new bone formation was evident in defects implanted with $\mathrm{mSIS} / \mathrm{P} 28$ and mSIS membranes. Further analysis at higher magnification showed that mSIS/P28 group possessed several larger bony nodules, which led to the formation of thin strips of bony tissue (Figure 9F). Typical histologic characteristics of newly formed bone were observed, with osteocytes embedded in the lacuna and osteoblasts lining the outer edge of the bone tissue (Figure 9F).

The results of the histomorphometric assay are shown in Figure 9E. Eight weeks after surgery, the percentage of new bone area in mSIS/P28 and mSIS groups was $38.94 \% \pm 5.26 \%$ and $16.27 \% \pm 3.89 \%$, respectively, which was significantly higher $(p<0.05)$ than that reported for SIS group $(2.14 \% \pm 1.03 \%)$. In addition, a significant difference was observed in the new bone area percentage between mSIS/P28 and mSIS groups, as observed with micro-CT analysis. These results suggest that the mSIS membrane promoted bone regeneration in an osteoporotic defect and that the incorporation of P28 into mSIS further enhanced its stimulatory effect on osteogenesis.

\section{Discussion}

An ideal GBR membrane should be biodegradable and biocompatible and should exhibit space-maintaining and bone regeneration abilities as well as clinical manageability. ${ }^{35}$ At present, several composites of degradable membranes such as polytetrafluoroethylene, collagen membrane, HA/poly L-lactide, and poly L-lactide/alginate have been developed. ${ }^{5,36}$ However, these membranes lack the characteristics of
A

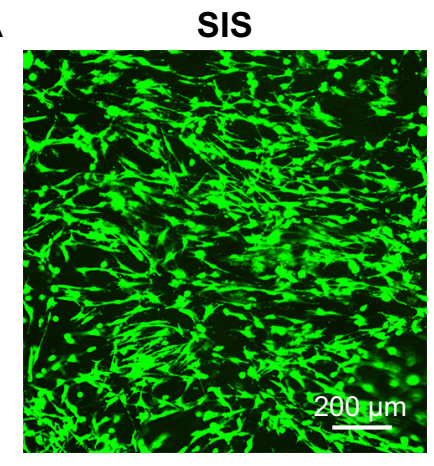

mSIS

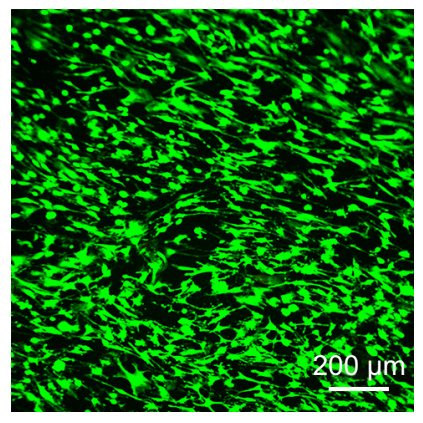

mSIS/P28

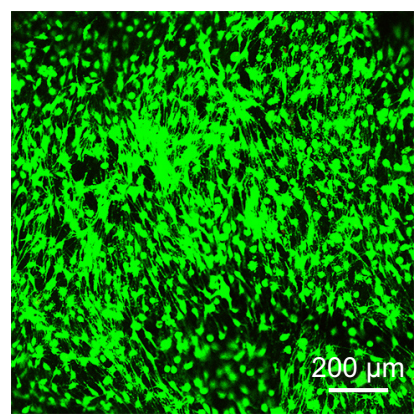

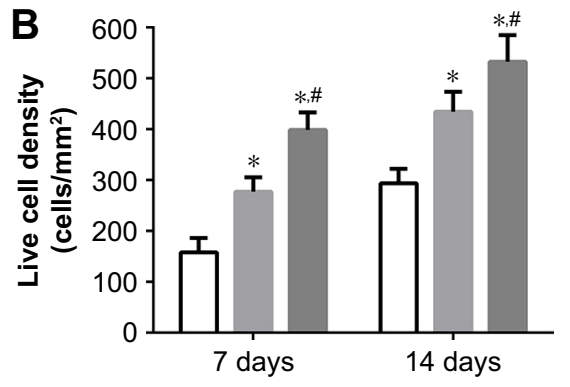

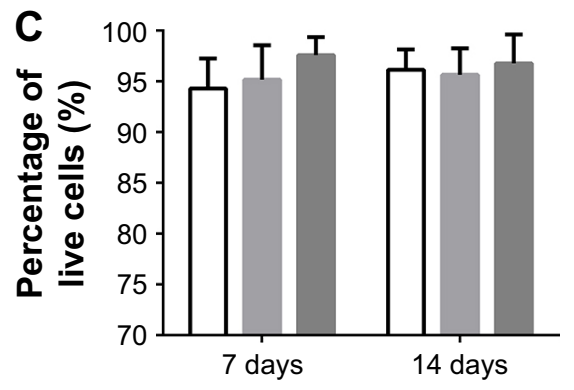

SIS

mSIS

$\mathrm{mSIS} / \mathrm{P} 28$

Figure 6 Live/dead assay of rBMSCs-OVX cells on SIS, mSIS, and mSIS/P28 membranes.

Notes: (A) Representative confocal images of cells on membranes at day 14. (B) Live cell density (number of live cells per mm²). (C) Percentage of live cells. Statistical significance is indicated by $* p<0.05$ compared to pure SIS group and ${ }^{*} p<0.05$ compared to mSIS group.

Abbreviations: mSIS, mineralized SIS; OVX, ovariectomy; rBMSCs-OVX, bone marrow stromal stem cells from ovariectomized rats; SIS, small intestinal submucosa. 
A

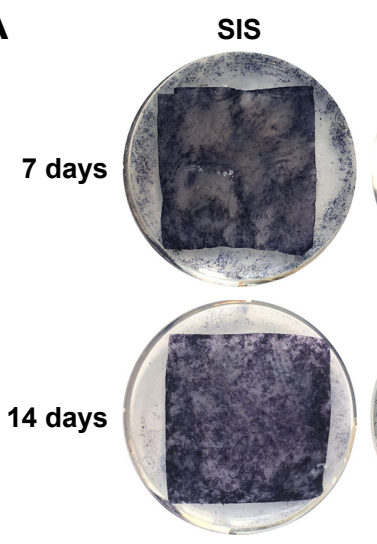

mSIS

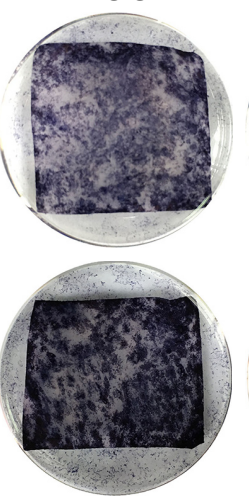

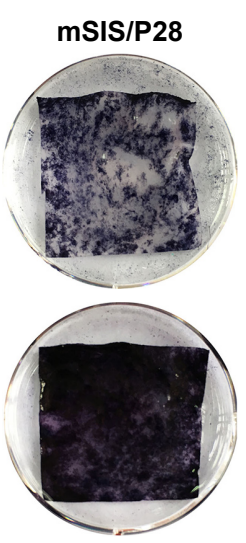

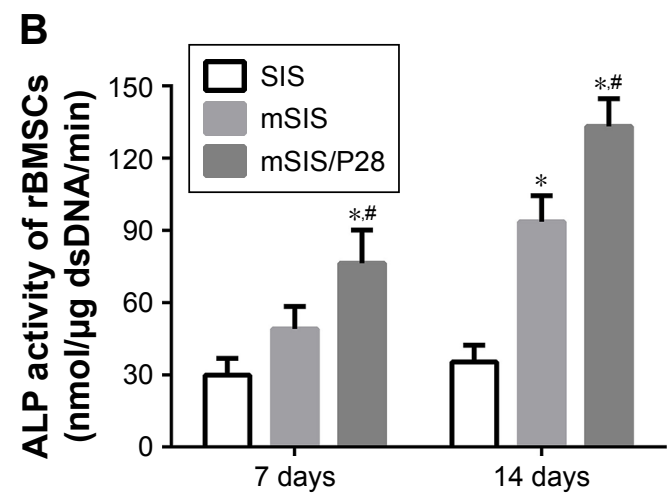

Figure 7 Osteogenic differentiation analysis.

Notes: (A) ALP staining of rBMSCs-OVX cells on SIS, mSIS, and mSIS/P28 scaffolds at days 7 and I4. (B) The quantification of ALP activity in cells on membranes at days 7 and 14. Statistical significance is indicated by $* p<0.05$ compared to pure SIS group and ${ }^{\#} p<0.05$ compared to mSIS group.

Abbreviations: ALP, alkaline phosphatase; mSIS, mineralized SIS; OVX, ovariectomy; rBMSCs-OVX, bone marrow stromal stem cells from ovariectomized rats; SIS, small intestinal submucosa.

GBR membrane, including the bone regeneration ability. In this study, a bioactive membrane was developed for guided osteoporotic bone regeneration by introducing a short BMP2-related peptide, P28, into heparinized mSIS.
The mSIS-heparin-P28 system was designed to improve the efficacy of P28 immobilization on mSIS and facilitate its controlled release. The results reveal that mSIS provides a favorable platform to load P28 as well as for its controlled
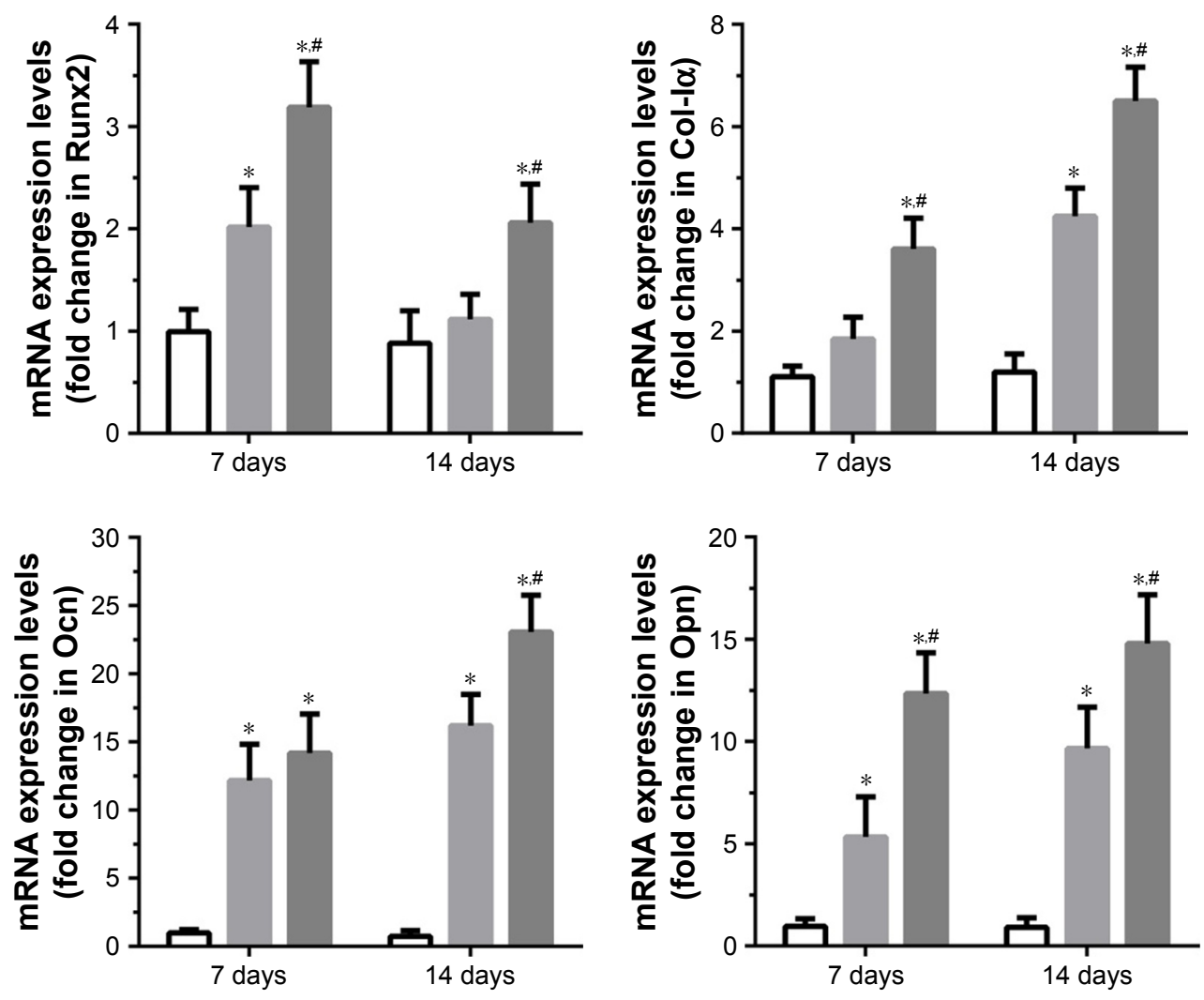

\section{$\square \mathrm{SIS}$ mSIS $\mathrm{mSIS/P28}$}

Figure 8 Time course changes in mRNA expression of osteogenic markers, such as Runx2, Col-l $\alpha$, Ocn, and Opn, in rBMSCs-OVX cells cultured on SIS, mSIS, and mSIS/ P28 membranes for 7 and 14 days.

Note: Statistical significance is indicated by ${ }^{*} p<0.05$ compared to pure SIS group and ${ }^{*} p<0.05$ compared to mSIS group.

Abbreviations: mSIS, mineralized SIS; OVX, ovariectomy; rBMSCs-OVX, bone marrow stromal stem cells from ovariectomized rats; SIS, small intestinal submucosa. 
A
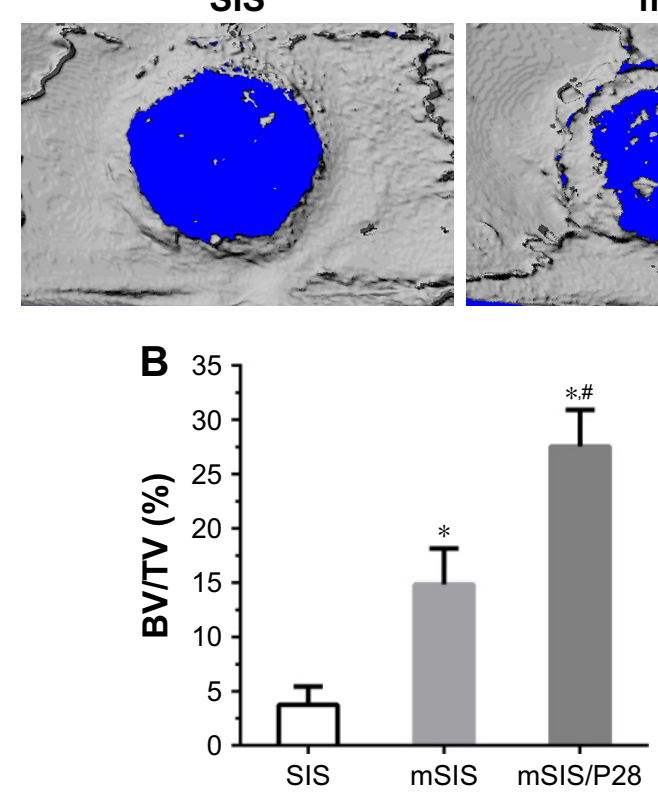

D
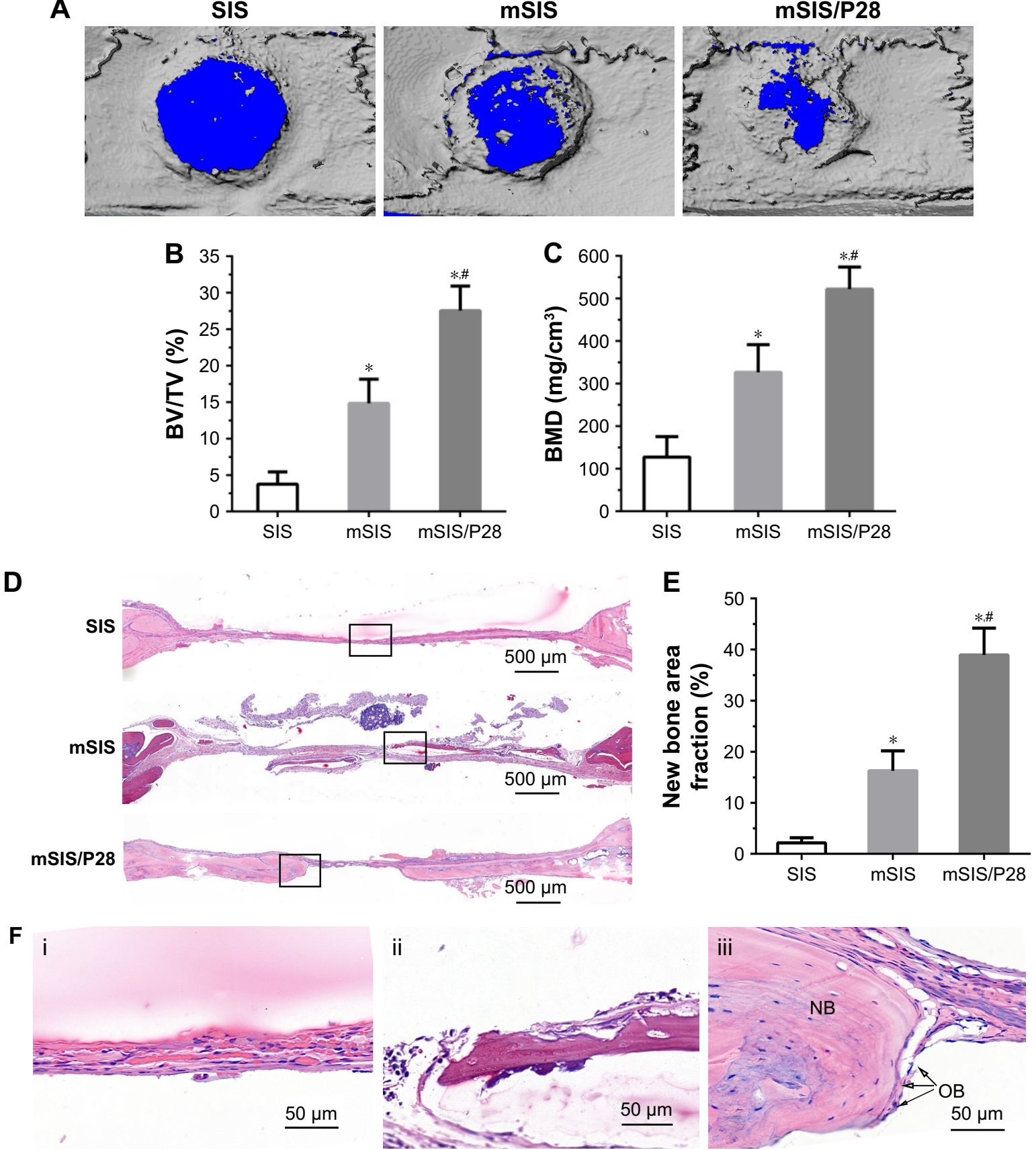

Figure 9 Evaluation of bone regeneration in calvarial defects of ovariectomized rats.

Notes: (A) Representative micro-CT images of calvarial defects treated with SIS, mSIS, and mSIS/P28 membranes. (B and C) BV/TV and BMD in the cranial defect implanted with those membranes. (D) Representative H\&E staining images of SIS, mSIS, and mSIS/P28 groups at 8 weeks postsurgery. (F) High magnification images of the black rectangles in D (i, SIS; ii, mSIS; and iii, mSIS/P28). The osteoblast and new bone are indicated by the abbreviations "OB" and "NB", respectively. (E) Histomorphometry analysis of percentage of new bone area in the rat cranial defect. Statistical significance is indicated by $* p<0.05$ compared to pure SIS group and ${ }^{\#}<0.05$ compared to mSIS group. Abbreviations: BMD, bone mineral density; BV, bone volume; micro-CT, micro-computed tomography; mSIS, mineralized SIS; SIS, small intestinal submucosa; TV, tissue volume.

release, and the combination of mSIS and P28 enhances osteoporotic bone regeneration by stimulating the proliferation and osteogenic differentiation of rBMSCs-OVX. Thus, $\mathrm{mSIS} / \mathrm{P} 28$ proved to be an ideal GBR membrane for guided osteoporotic bone regeneration.

Although SIS possesses an excellent microenvironment to guide cell response, its composition and architecture are far different from those of the natural bone. Therefore, it is necessary to tailor its composition and architecture for improved osteoinductivity. Inspired by the process of calcium phosphate deposition within the collagenous matrix for bone development, ${ }^{37,38}$ several studies have attempted to develop bone-like ECM with the process of biomineralization. ${ }^{39,40}$ In this study, an HA/SIS membrane was fabricated by 
incubating SIS in SBF solution for biomineralization. SIS membrane mainly comprised collagen fibers (Figure 1). The incubation of the SIS membrane in SBF for a defined period resulted in the process of nucleation and crystals were deposited on SIS fibers. EDS and XRD analysis identified these crystals to be HA. XRD-specific peaks for HA were gradually enhanced with an increase in incubation time. This observation was in accordance with the gradual increase in the crystal size observed by SEM.

Various surface modification technologies are being explored to enhance the immobilization efficacy and sustained delivery of growth factors. Approaches of physical adsorption or direct covalent modification pose challenges such as low loading efficiency, burst release in a short time, or loss of the bioactivity of growth factors owing to covalent reactions. ${ }^{21}$ Heparin-functionalized scaffolds are known to enhance sustained release of growth factors and decrease loss in bioactivity as compared with the scaffold covalently modified with $N$-(3-dimethylaminopropyl)- $N^{\prime}$ ethylcarbodiimide hydrochloride/ $N$-hydroxysuccinimide chemistry. ${ }^{41}$ In this study, heparin-functionalized mSIS was designed to improve the efficacy of P28 immobilization on $\mathrm{mSIS}$ and facilitate its long-term release. The in vitro release curve showed that P28 was initially released from heparinfunctionalized mSIS in a small burst, followed by a slow and sustained release maintained over time. This observation may be attributed to two types of affinities: the ionic bond between heparin and P28 and the high affinity of the seven Asp in P28 for HA deposited on mSIS membranes. In our previous study, P28 showed a more controlled release profile from an HA-incorporated scaffold as compared to the scaffold without HA. ${ }^{17,18}$ By day 21, P28 was almost completely released $(98.35 \%)$ from $\mathrm{mSIS}$ without heparinization. On the contrary, a local and sustained delivery of P28 from heparinized mSIS was observed, with a long-term release time of almost 40 days.

The osteoblastic differentiation ability of bone marrow stromal cells (BMSCs) derived from osteoporotic patients is significantly reduced, ultimately leading to a delay in bone formation or nonunion. ${ }^{1}$ The OVX rat model has been validated as a clinically relevant model of human postmenopausal bone loss and is commonly used to investigate the pathophysiology, diagnosis, and therapy of osteoporosis. ${ }^{42}$ As shown in Figure 4, micro-CT scanning showed that the BV was significantly reduced after ovariectomy, while ARS staining revealed decrease in the osteogenic ability of BMSCs from OVX. Our previous studies have shown that P28 successfully stimulated osteogenic differentiation of BMSCs from healthy tissues in vitro and enhanced bone regeneration in normal animals in vivo. ${ }^{27}$ This is the first report to explore the biologic effect of $\mathrm{P} 28$ under osteoporotic pathologic condition.

The cell response to biomaterials depends not only on the chemical composition but also on the surface characteristics such as stiffness and roughness. ${ }^{43}$ The mineralization treatment of SIS led to HA crystal deposition, thereby imparting a rough and rigid surface to SIS. HA is proved to promote osteogenic differentiation of stem cells, ${ }^{18}$ and a rigid and rough matrix may promote cell differentiation toward osteogenic lineage. ${ }^{44,45}$ In this study, MTT assay demonstrated that mSIS with HA crystal deposition significantly promoted rBMSCs-OVX proliferation as compared with pure SIS. In comparison with pure SIS, mSIS significantly elevated ALP activity and the expression of osteogenic-related genes without additional osteogenic components. Moreover, the incorporation of P28 into mSIS resulted in a higher stimulatory effect on the proliferation and osteogenic differentiation of BMSCs-OVX as compared with mSIS without P28, suggestive of the synergistic effect of P28 and mSIS on the osteogenesis activity.

To explore the potential clinical applications of mSIS/ P28 in osteoporotic patients, a critical-sized OVX calvarial defect model was used. ${ }^{29}$ In vivo results demonstrated that $\mathrm{mSIS} / \mathrm{P} 28$ and mSIS enhanced osteoporotic bone regeneration as compared with pure SIS in OVX rat calvarial defects and that $\mathrm{mSIS} / \mathrm{P} 28$ induced the highest bone regeneration. Micro-CT analysis showed a higher BV/TV ratio indicative of new bone fraction for $\mathrm{mSIS} / \mathrm{P} 28$ and $\mathrm{mSIS}$ groups as compared with pure SIS group; mSIS/P28 group showed the highest ratio. The results of the histologic analysis coincided with the findings of micro-CT scan. Thus, P28 released from mSIS possessed a strong osteoinductive bioactivity in vivo and mSIS/P28 enhanced the formation of new bone and accelerated the healing of critical-sized bone defects in the osteoporosis model. Although the scaffold was derived from porcine jejunum, we failed to observe any obvious immunologic response and inflammatory signs, indicative of successful decellularization. Taken together, mSIS enhanced the regeneration of osteoporotic bone defects as compared with pure SIS and the combination of mSIS and BMP2-related peptide further improved its bone regeneration efficacy.

\section{Conclusion}

In this study, a novel GBR membrane, $\mathrm{mSIS} / \mathrm{P} 28$, was fabricated for guided osteoporotic bone regeneration. Heparin-functionalized mSIS was designed to improve the efficacy of P28 immobilization on mSIS and facilitate its long-term release. In vitro results revealed P28 release in a 
spatiotemporal control model from the mSIS-heparin-P28 system, and the synergistic stimulation by both mSIS and P2 8 provided a preferential environment for inducing rBMSCsOVX differentiation toward osteoblasts. In addition, the osteoporotic bone regeneration in a critical-sized calvarial defect OVX rats showed that mSIS/P28 has a superior osteoinductive activity to enhance early bone formation as compared with mSIS. Our study demonstrates the promising application of mSIS/P28 as a GBR membrane for the regeneration of osteoporotic bone defects.

\section{Acknowledgments}

This work was supported by the National Natural Science Foundation of China (grant numbers 81672158 and 81371939) and the National Key R\&D Program of China (2016YFC1100100).

\section{Disclosure}

The authors report no conflicts of interest in this work.

\section{References}

1. Li KC, Chang YH, Yeh CL, Hu YC. Healing of osteoporotic bone defects by baculovirus-engineered bone marrow-derived MSCs expressing MicroRNA sponges. Biomaterials. 2016;74:155-166.

2. Xia L, Yin Z, Mao L, et al. Akermanite bioceramics promote osteogenesis, angiogenesis and suppress osteoclastogenesis for osteoporotic bone regeneration. Sci Rep. 2016;6:22005.

3. Guda T, Walker JA, Singleton BM, et al. Guided bone regeneration in long-bone defects with a structural hydroxyapatite graft and collagen membrane. Tissue Eng Part A. 2013;19(17-18):1879-1888.

4. Elgali I, Turri A, Xia W, et al. Guided bone regeneration using resorbable membrane and different bone substitutes: early histological and molecular events. Acta Biomater. 2016;29:409-423.

5. Piao ZG, Kim JS, Son JS, et al. Osteogenic evaluation of collagen membrane containing drug-loaded polymeric microparticles in a rat calvarial defect model. Tissue Eng Part A. 2014;20(23-24):3322-3331.

6. Brown BN, Badylak SF. Extracellular matrix as an inductive scaffold for functional tissue reconstruction. Transl Res. 2014;163(4):268-285.

7. Badylak SF, Record R, Lindberg K, Hodde J, Park K. Small intestinal submucosa: a substrate for in vitro cell growth. J Biomater Sci Polym Ed. 1998;9(8):863-878.

8. Hodde JP, Badylak SF, Brightman AO, Voytik-Harbin SL. Glycosaminoglycan content of small intestinal submucosa: a bioscaffold for tissue replacement. Tissue Eng. 1996;2(3):209-217.

9. Lin X, Robinson M, Petrie T, Spandler V, Boyd WD, Sondergaard CS. Small intestinal submucosa-derived extracellular matrix bioscaffold significantly enhances angiogenic factor secretion from human mesenchymal stromal cells. Stem Cell Res Ther. 2015;6(1):164.

10. Hodde J, Janis A, Ernst D, Zopf D, Sherman D, Johnson C. Effects of sterilization on an extracellular matrix scaffold: part I. Composition and matrix architecture. J Mater Sci Mater Med. 2007;18(4):537-543.

11. Turri A, Elgali I, Vazirisani F, et al. Guided bone regeneration is promoted by the molecular events in the membrane compartment. Biomaterials. 2016;84:167-183.

12. Yao S, Jin B, Liu Z, et al. Biomineralization: from material tactics to biological strategy. Adv Mater. 2017;29(14).

13. Lee JC, Volpicelli EJ. Bioinspired collagen scaffolds in cranial bone regeneration: from bedside to bench. Adv Healthc Mater. 2017;6(17).

14. Zimmermann EA, Ritchie RO. Bone as a structural material. Adv Healthc Mater. 2015;4(9):1287-1304.
15. Liu Y, Liu S, Luo D, et al. Hierarchically staggered nanostructure of mineralized collagen as a bone-grafting scaffold. Adv Mater. 2016; 28(39):8740-8748.

16. Carreira AC, Lojudice FH, Halcsik E, Navarro RD, Sogayar MC, Granjeiro JM. Bone morphogenetic proteins: facts, challenges, and future perspectives. J Dent Res. 2014;93(4):335-345.

17. Cui W, Sun G, Qu Y, et al. Repair of rat calvarial defects using Si-doped hydroxyapatite scaffolds loaded with a bone morphogenetic protein-2related peptide. J Orthop Res. 2016;34(11):1874-1882.

18. Sun T, Zhou K, Liu M, et al. Loading of BMP-2-related peptide onto three-dimensional nano-hydroxyapatite scaffolds accelerates mineralization in critical-sized cranial bone defects. J Tissue Eng Regen Med. Epub 2016 Nov 25.

19. Hecker $A$, Testenière $O$, Marin $F$, Luquet G. Phosphorylation of serine residues is fundamental for the calcium-binding ability of Orchestin, a soluble matrix protein from crustacean calcium storage structures. FEBS Lett. 2003;535(1-3):49-54.

20. Kasugai S, Fujisawa R, Waki Y, Miyamoto K, Ohya K. Selective drug delivery system to bone: small peptide (Asp)6 conjugation. $J$ Bone Miner Res. 2000;15(5):936-943.

21. Knaack S, Lode A, Hoyer B, et al. Heparin modification of a biomimetic bone matrix for controlled release of VEGF. J Biomed Mater Res A. 2014;102(10):3500-3511.

22. Ferreira AM, Gentile P, Toumpaniari S, Ciardelli G, Birch MA. Impact of collagen/heparin multilayers for regulating bone cellular functions. ACS Appl Mater Interfaces. 2016;8(44):29923-29932.

23. Zwingenberger S, Langanke R, Vater C, et al. The effect of SDF-1alpha on low dose BMP-2 mediated bone regeneration by release from heparinized mineralized collagen type I matrix scaffolds in a murine critical size bone defect model. J Biomed Mater Res A. 2016; 104(9):2126-2134.

24. Luo JC, Chen W, Chen XH, et al. A multi-step method for preparation of porcine small intestinal submucosa (SIS). Biomaterials. 2011; 32(3):706-713.

25. Wu C, Zhang Y, Zhou Y, Fan W, Xiao Y. A comparative study of mesoporous glass/silk and non-mesoporous glass/silk scaffolds: physiochemistry and in vivo osteogenesis. Acta Biomater. 2011;7(5): 2229-2236.

26. Yang HS, La WG, Bhang SH, Jeon JY, Lee JH, Kim BS. Heparinconjugated fibrin as an injectable system for sustained delivery of bone morphogenetic protein-2. Tissue Eng Part A. 2010;16(4):1225-1233.

27. Sun T, Qu Y, Cui W, et al. Evaluation of osteogenic inductivity of a novel BMP2-mimicking peptide P28 and P28-containing bone composite. J Biomed Mater Res A. 2017;106(1):210-220.

28. Liu Y, Min L, Luo H, et al. Integration of a calcined bovine bone and BMSC-sheet 3D scaffold and the promotion of bone regeneration in large defects. Biomaterials. 2013;34(38):9998-10006.

29. Lin K, Xia L, Li H, et al. Enhanced osteoporotic bone regeneration by strontium-substituted calcium silicate bioactive ceramics. Biomaterials. 2013;34(38):10028-10042.

30. Sun T, Yao S, Liu M, et al. Composite scaffolds of mineralized natural extracellular matrix on true bone ceramic induce bone regeneration via Smad1/5/8 and ERK1/2 pathways. Tissue Eng Part A. Epub 2017 Aug 15.

31. Zurick KM, Qin C, Bernards MT. Mineralization induction effects of osteopontin, bone sialoprotein, and dentin phosphoprotein on a biomimetic collagen substrate. J Biomed Mater Res A. 2013;101(6):1571-1581.

32. Li J, Zheng Q, Guo X, et al. Bone induction by surface-doublemodified true bone ceramics in vitro and in vivo. Biomed Mater. 2013; 8(3):035005

33. Si J, Cui Z, Wang Q, Liu Q, Liu C. Biomimetic composite scaffolds based on mineralization of hydroxyapatite on electrospun poly(varepsilon-caprolactone)/nanocellulose fibers. Carbohydr Polym. 2016;143:270-278.

34. Cheng N, Wang Y, Zhang Y, Shi B. The osteogenic potential of mesoporous bioglasses/silk and non-mesoporous bioglasses/silk scaffolds in ovariectomized rats: in vitro and in vivo evaluation. PLoS One. 2013; 8(11):e81014. 
35. Ma S, Adayi A, Liu Z, et al. Asymmetric collagen/chitosan membrane containing minocycline-loaded chitosan nanoparticles for guided bone regeneration. Sci Rep. 2016;6:31822.

36. Ferreira AM, Gentile P, Chiono V, Ciardelli G. Collagen for bone tissue regeneration. Acta Biomater. 2012;8(9):3191-3200.

37. Nudelman F, Lausch AJ, Sommerdijk NA, Sone ED. In vitro models of collagen biomineralization. J Struct Biol. 2013;183(2):258-269.

38. Berendsen AD, Olsen BR. Bone development. Bone. 2015;80:14-18.

39. Cui FZ, Li Y, Ge J. Self-assembly of mineralized collagen composites. Mater Sci Eng R. 2007;57(1-6):1-27.

40. Ren X, Bischoff D, Weisgerber DW, et al. Osteogenesis on nanoparticulate mineralized collagen scaffolds via autogenous activation of the canonical BMP receptor signaling pathway. Biomaterials. 2015; 50:107-114.

41. Wu JM, Xu YY, Li ZH, et al. Heparin-functionalized collagen matrices with controlled release of basic fibroblast growth factor. J Mater Sci Mater Med. 2011;22(1):107-114.
42. Ezirganli S, Kazancioglu HO, Acar AH, Özdemir H, Kuzu E, İnan DŞ. Effects of Ankaferd BloodStopper on bone healing in an ovariectomized osteoporotic rat model. Exp Ther Med. 2017;13(5):1827-1831.

43. Gupta P, Adhikary M, M JC, Kumar M, Bhardwaj N, Mandal BB. Biomimetic, osteoconductive non-mulberry silk fiber reinforced tricomposite scaffolds for bone tissue engineering. ACS Appl Mater Interfaces. 2016;8(45):30797-30810.

44. Reilly GC, Engler AJ. Intrinsic extracellular matrix properties regulate stem cell differentiation. J Biomech. 2010;43(1):55-62.

45. Khatiwala CB, Peyton SR, Metzke M, Putnam AJ. The regulation of osteogenesis by ECM rigidity in MC3T3-E1 cells requires MAPK activation. J Cell Physiol. 2007;211(3):661-672.

\section{Publish your work in this journal}

The International Journal of Nanomedicine is an international, peerreviewed journal focusing on the application of nanotechnology in diagnostics, therapeutics, and drug delivery systems throughout the biomedical field. This journal is indexed on PubMed Central, MedLine, CAS, SciSearch $\AA$, Current Contents ${ }^{\circledR} /$ Clinical Medicine,
Journal Citation Reports/Science Edition, EMBase, Scopus and the Elsevier Bibliographic databases. The manuscript management system is completely online and includes a very quick and fair peer-review system, which is all easy to use. Visit http://www.dovepress.com/ testimonials.php to read real quotes from published authors. 